\title{
Tangence
}

\section{L'inquiétante familiarité}

\section{Jean-Pierre Vidal}

Numéro 52, septembre 1996

Tours et détours du romanesque : Minuit aujourd'hui

URI : https://id.erudit.org/iderudit/025913ar

DOI : https://doi.org/10.7202/025913ar

Aller au sommaire du numéro

Éditeur(s)

Tangence

ISSN

0226-9554 (imprimé)

1710-0305 (numérique)

Découvrir la revue

Citer cet article

Vidal, J.-P. (1996). L'inquiétante familiarité. Tangence, (52), 9-24.

https://doi.org/10.7202/025913ar d'utilisation que vous pouvez consulter en ligne.

https://apropos.erudit.org/fr/usagers/politique-dutilisation/ 


\section{L'inquiétante familiarité}

Jean-Pierre Vidal

Comme chez le Robbe-Grillet deuxième manière, celui qui commence avec La maison de rendez-vous, ou même, sous l'angle de l'expansion variationnelle à partir d'une cellulé scripturale minimale, déjà avec Dans le labyrintbe, il y a du picaresque dans les romans de Jean-Philippe Toussaint. Non pas celui, exubérant et rigolard du modèle historique qui déploie complaisamment, comme au fil de la plume, d'extravagantes aventures, mais un picaresque pince-sans-rire qui tient à un enchaînement imperturbable d'événements banals produits par quelque clinamen au coeur du quotidien: la décision soigneusement injustifiée mais assez explicitement kafkaïenne de s'installer dans sa baignoire (La salle de bain), la décision, encore, d'apprendre à conduire, coincidant avec le mariage d'un ami perdu de vue et tout ce qui découle du manque de photos nécessaires au dossier ouvert par l'auto-école (L'appareil-pboto), enfin une hésitation à aller surprendre à l'improviste des amis italiens (La réticence). Une exception: Monsieur où, loin qu'il y ait, dès l'ouverture et par la suite, l'action de quelque proverbial grain de sable, tout, au contraire, d'entrée de jeu, "baigne" et jusqu'à l'exclamation finale: "La vie, pour Monsieur, un jeu d'enfant" 1 . Encore, dans ce dernier cas, une incise nous prévient-elle, dès l'incipit, de quelque inquiétude sourde que le narrateur attribue au personnage, dans une fort curieuse focalisation qui prend la forme d'une analepse sertie dans la narration comme une pseudo-citation au style indirect libre, premier accroc presque invisible à la trame narrative: :Le jour où, voici trois ans, Monsieur entra dans ses nouvelles fonctions, on lui attribua un bureau personnel, jusqu'à présent c'était parfait, au seizième étage, tour Léonard-de-Vincin (p. 7).

- Quoi qu'il en soit, hormis le cas particulier de ce deuxième roman qui forme comme une version euphorique de la fabulation selon Toussaint, les trois autres romans à ce jour publiés, proposent tous une variation sur l'accidentel assumé et la dialectique entropie-négentropie qui effrite, reforme, défait, reconstruit

1 Monsteur, Paris, Editions de Minuit, 1986, p. 111. 
encore, en une sorte de chaîne sans fin, le modeste parcours événementiel de ses falots héros.

Sans doute est-ce pour cette raison, cette obsession d'abord scripturale, au mitan de laquelle La salle de bain inscrit d'ailleurs en toutes lettres le nombre pythagoricien, concentré de symbolique et de code, que l'œuvre de Toussaint, telle qu'elle est, pour le moment, constituée, s'avère étonnamment circulaire, La salle de bain et La réticence encerclant d'une poétique "pascalienne" de l'immobilité contrariée les deux romans médians qui, eux, semblent plutôt mettre en scène la fuite en avant et le phénomène de relais, encore que l'importance de l'installation, de l'occupation heureuse de l'espace de Monsieur puisse représenter la version "positive" de l'enfouissement "tragique ", et tout uniment humoristique, de ces deux derniers. Le personnage central de Monsieur, en effet, prend ses aises, est l'aise même, partout, en tout, inatteignable dans sa tour, alors que celui de La salle de bain, par exemple, ne rêve que d'engloutissement ${ }^{2}$, et se déplace, symptomatiquement, d'un espace sanitaire à un espace hospitalier. C'est pourquoi, sans doute, Monsieur est le seul des quatre romans à offrir une narration à la troisième personne: celui qu'une ironie nominale fait trôner sous le titre "Monsieur " 3 ,

2 Il prétend même aider à la submersion de Venise en sautant à pieds joints sur ses pavés ( La salle de bain, Paris, Éditions de Minuit, 1985, p. 81) et, en guise d'histoire, entreprend de raconter à la fillette de son médecin le naufrage du Titanic (ibid., p. 104).

3 Fût-il ironiquement *titré *, il n'en rejoint pas moins dans l'anonymat total les trois autres personnages principaux, réduits tout uniment à des * je *, privilège certes attaché au narrateur homodiégétique mais qui se souligne ici comme un parti pris signifiant supplémentaire, dans la mesure où les trois textes multiplient les occasions de décliner son identité (passage de frontières, hôtels, hôpital). Le silence du nom propre est donc sans doute en rapport avec une position ontologique et fait partie d'un système onomastique particulièrement retors qui, pour ne prendre, faute de temps, que l'exemple de La salle de bain, articule le nom propre (et même le prénom) tu du narrateur au patronyme tonitruant de sa compagne appelée, contre toute attente "réaliste •, exclusivement *Edmondsson *, à ceux des peintres polonais, plus polonais qu'il n'est raisonnable et rimant entre eux (*Kabrowinski * et *Kovalskazinski *, ce dernier improbablement prénommé Jean-Marie quand l'autre reste un bon vieux Witold - hommage à Gombrowicz dont Toussaint n'est pas littérairement si éloigné?), au nom expressément imaginé de l'ambassadeur d'Autriche, Eigenschaften - on ne saurait faire plus ironique, en guise de nom propre, que le substantif pluriel qui en allemand veut dire, justement, -qualités, propriétés»; l'ambassadeur est, en quelque sorte, Monsieur paradigme ou Monsieur sujet, ce qui donne un sens bien particulier à cette 
avec majuscule, est décidément l'Autre, celui dont on ne peut jamais saisir le malaise, imaginer les états d'âme et de conscience, celui qu'on observe avec amusement, comme un insecte social rare. Sans doute aussi est-ce pour cette raison que ce roman ne présente, au départ de la fiction, aucune rupture dans la continuité quotidienne, hormis celle, implicite et incontestablement peu douloureuse, qui consiste à occuper une nouvelle fonction.

Toujours pour traiter de l'opposition là où elle est la plus forte, c'est-à-dire entre les deux premiers romans qui forment ainsi décidément système, posant en quelque sorte les pôles entre lesquels les deux autres romans viendront s'inscrire, disposition bien entendu provisoire puisque l'œuvre est en cours, La salle de bain est au contraire le roman du discontinu, de l'alternance décision-dérive, ordre-désordre, fixité-mouvement. L'obsession entropie-négentropie qui lui donne son rythme narratif et scriptural évoque même parfois le Sartre de La nausée. En effet, comme Roquentin qui y voyait "la nature sans les hommes", le héros contemple à maintes reprises son visage, comme une énigme, dans la glace ou dans la transparence aveugle d'une radiographie, et la plus célèbre racine de toute l'histoire de la littérature n'est pas sans resurgir à la mémoire dans telle description hallucinée d'un innocent dessert livré à l'anaphore du regard, redit comme une scansion:

invitation de l'ambassade d'Autriche adressée à notre anonyme narrateur; et encore aux noms propres échangés avec le barman de l'hôtel dans une scène irrésistible de drôlerie: "L'absence d'une langue commune ne nous décourageait pas; sur le cyclisme, par exemple, nous étions intarissables. Moser disait-il. Merckx, faisais-je remarquer au bout d'un petit moment. Coppi, disait-il, Fausto Coppi. Je toumais ma cuillère dans le café, approuvant de la tête, pensif. Bruyère, murmurais-je. Bruyère? disait-il. Oui, oui, Bruyère. Il ne semblait pas convaincu. Je pensais que la conversation s'en tiendrait là, mais, alors que je me disposais à quitter le comptoir, me retenant par le bras, il m'a dit Gimondi. Van Springel, répondis-je. Planckaert, ajoutaije, Dierieckx, Willems, Van Impe, Van Looy, de Vlaeminck, Roger de Vlaeminck et son frère, Eric. Que pouvait-on répondre à cela? Il n'insista pas. * (p. 61-62). Avec le patronyme pléthorique et cycliste de l'Autre, c'est la patrie qui s'échange et se décline puisque le narrateur est belge, comme les champions qu'il oppose à ceux du barman italien. Ici l'onomastique rejoint la traduction - $c f$., plus loin, dans cet article - puisque, détail non négligeable, à part le fort improbable Bruyère (que de plus fins connaisseurs que nous ès science vélocipédique nous'détrompent!), tous ces chevaliers de "la petite reine. sont des Flamands et que le narrateur, jusqu'à plus ample informé s'exprime (narre? écrit?) en français. 
Je regardais la dame blanche fondre devant moi. Je regardais fondre imperceptiblement la vanille sous la nappe de chocolat brûlant. Je regardais la boule encore exactement ronde un instant plus tôt qui ruisselait lentement en filets réguliers blancs et bruns métissés. Je regardais le mouvement, immobile, les yeux fixés sur la soucoupe. Je ne bougeais pas. Les mains figées sur la table, j'essayais de toutes mes forces de garder l'immobilité, de la retenir, mais je sentais bien que, sur mon corps aussi, le mouvement s'écoulait. ${ }^{4}$

"Je regardais le mouvement, immobile [...] j'essayais de toutes mes forces de garder l'immobilité, de la retenir...", on voit clairement que le drame est ontologique et l'obsession phénoménologique ${ }^{5}$ : aller aux choses, $y$ fondre son regard mais pour mieux asseoir la permanence de son extériorité, appréhender leur immobilité, leur silence qui sans cesse nous survit avec fracas, et les voir elles aussi s'éroder, passer, muer, mourir, dans le flux du mouvement qui nous aussi, qui nous surtout, nous point et nous efface, insensiblement. C'est l'expérience proprement philosophique, heideggerienne même, sur laquelle se fonde la poétique du roman moderne, telle que Sartre l'a magistralement amorcée en France, dès 1938, avec La nausée, avant que le Nouveau Roman ${ }^{6}$ n'en fasse son véritable fondement idéologique et scriptural: l'écriture comme balisage de la distance des mots aux choses et de l'homme aux mots, ce qu'on appelle, somme toute, le référent, tenon du signifiant bien plus qu'،image" réelle du signifié "vir-

$4 \quad$ Ibid, p. 80.

5 Le sens qui déjouait la contingence chez Sartre, on s'en souviendra, était représenté par l'air de jazz entendu au café, le jazz, art populaire et nègre, art d'improvisation mais dont l'ordre paraît soudain imparable à Roquentin. Il semblerait qu'ici le sens comme salut soit représenté, pour le narrateur, non pas par l'art - ça, c'est surtout l'affaire d'Edmondsson, encore que le * je * en tâte aussi - mais par le sport, avec ses règles et ses cérémonies : football et cyclisme professionnels, sports de masse dont on n'est que spectateur, tennis, sport encore vaguement élitiste auquel on ne parvient pas à jouer, et surtout les fléchettes, sur lesquelles je reviendrai, sport de chambre, sport anodin mais devenu ici fort dangereux. Et qui, curieusement, je tenterai de le montrer, rejoint l'art.

6 Pour la parenté, dans l'articulation description-fiction *ontologique ", entre La nausée et le Nouveau Roman, $c f$. mon * Bretelles mauves, mains rouges et mur chocolat: les couleurs de La nausée*, Protée, vol II, no 1, avril 1972, p. 73-84. Georges Raillard a d'ailleurs dans d'autres contextes et à d'autres propos, signalé lui aussi, à plusieurs reprises, cette place de choix prise par La nausée dans les textes en quelque sorte fondateurs de ce qui allait devenir le Nouveau Roman. 
tuel" à quoi on le réduit bien souvent. C'est par le référent en effet que le signifiant peut faire sonner sa chaîne comme une musique et se mettre ainsi en évidence comme un hors-lieu qui dit le lieu de l'homme, selon l'incontoumable leçon de Mallarmé. Car le signifiant est toujours un saut ${ }^{7}$, un grand écart entre deux abîmes, deux spectres inatteignables et inévitables: le sujet et le monde.

Cette quête signifiante, ce parcours "immobile à grands pas n, pour paraphraser Valéry, ce battement signifiant qui est l'émoi d'un regard, fût-il suspendu, l'explicit de L'appareil-photo lui donne une forme particulièrement forte et étonnamment proche de la "méditation" heideggerienne:

Je pensais oui, et lorsque je pensais, les yeux fermés et le corps à l'abri, je simulais une autre vie, identique à la vie dans ses formes et son souffle, sa respiration et son rythme, une vie en tous points comparable à la vie, mais sans blessure imaginable, sans agression et sans douleur possible, lointaine, une vie détachée qui s'épanouissait dans les décombres exténués de la réalité extérieure, et où une réalité tout autre, intérieure et docile, prenait la mesure de la douceur de chaque instant qui passait, et ce n'était guère des mots qui me venaient alors, ni des images, peu de sons si ce n'est le même murmure familier, mais des formes en mouvement qui suivaient leur cours dans mon esprit comme le mouvement même du temps, avec la même évidence infinie et sereine, formes tremblantes aux contours insaisissables que je laissais s'écouler en moi en silence dans le calme et la douceur d'un flux inutile et grandiose. ${ }^{8}$

L'autre vie, simulée par la pensée, est à la fois parallèle, de ce parallélisme ouvert par le symbolique sous les espèces de la mimesis, et intercalée, ponctuation dont l'imaginaire marque son emprise, toujours défaite et toujours reformée, sur le réel. Cette vie "s'épanouiss[ant] dans les décombres exténués de la réalité extérieure "est une sorte de point d'orgue où le sujet vient définir son lieu l'espace d'une variation *fractale * (*des formes en mouvement qui suivaient leur cours dans mon esprit *) et atteint ainsi à la conscience de ce qui fonde son rapport à l'être: le temps. C'était déjà, en somme, pour pouvoir laisser s'écouler en lui

7 Ou un lancer: les fléchettes de La salle de bain, avec leur téléologie d'abord solipsiste puis, au contraire, agressive, en sont peut-être la représentation la plus nette.

8 L'appareil-pboto, Paris, Éditions de Minuit, 1988, p. 125. 
14

"dans le calme et la douceur d'un flux inutile et grandiose " ces "formes tremblantes aux contours insaisissables" que le narrateur de La salle de bain tentait de transformer le sanitaire en thébaïde. Mais le paradoxe douloureux de cette "ouverture à l'être", comme dirait Heidegger encore, c'est qu'il tue. Les dernières lignes de L'appareil-pboto sont particulièrement éloquentes: "[...] je regardais le jour se lever et songeais simplement au présent, à l'instant présent, tâchant encore une fois de fixer sa fugitive grâce - comme on immobiliserait l'extrémité d'une aiguille dans le corps d'un papillon vivant. Vivant. "(p. 127).

Toute la manière de Toussaint est là, dans cette atténuation et cette ambiguitté; atténuation du percement de l'aiguille ${ }^{9}$ que l'on n'enfonce pas, que l'on ne fait pas pénétrer mais dont on «immobilise l'extrémité ", fin de course d'un corps qui en rencontre seulement un autre, métaphore de l'esprit épandu jusqu'à la saisie "phénoménologique" du monde. Ambiguité de la vie ainsi saisie, *épinglée * vive, comme on dit *brûlée vive ", et qui n'est donc saisie toujours que comme ayant eu lieu, le deuxième "vivant" qui clôt le texte formant une sorte de cri de triomphe ou d'extase où ce qui se proclame, c'est la littérature, espace propre de la vie qui tue et fait renaître, recherche dérisoire et magnifique du temps toujours déjà perdu, cérémonieuse avancée vers l'insaisissable dont l'écriture fait un paradoxal futur.

Dans La salle de bain où nous nous installons maintenant, l'expérience de ce qu'il faut bien appeler le sens, est, on l'a dit, placée sous l'invocation pythagoricienne du nombre, sous la forme d'un exergue ironique qui cite le fameux théorème sur le carré de l'hypoténuse. Elle est aussi pascalienne, et dans ce cas aussi en toutes lettres, non plus d'exergue cependant mais de citation, expressément, et tout aussi ironiquement encore parce qu'outrageusement en anglais la citation des Pensées!

Il est question ici, bien sûr, de savoir et de pouvoir * demeurer en repos ${ }^{10}$.

9 Avatar, sans nul doute, des fléchettes de La salle de bain qui, elles, finissent par * s'immobiliser • dans le corps d'Edmondsson. J'y reviendrai.

10 *...] j'ai découvert que tout le malheur des hommes vient d'une seule chose, qui est de ne savoir pas demeurer en repos, dans une chambre. "Blaise Pascal, Pensées, numéro 139 (-Divertissement *) de l'édition Brunschvicg, Éditions Garnier Frères, Paris 1958 [1649], p. 109. 


\section{L'installation dans le transitoire ou l'homme sans divertissement}

Comme en un rêve, les proches du narrateur de La salle de bain ne se montrent guère surpris de sa décision de camper en permanence dans sa baignoire: ils s'occupent, au contraire, à lui faciliter la vie, le visitent en audience, tournent autour de ce point fixe inattendu d'où notre anachorète, décidément bien mondain, ne rend pourtant avec superbe que l'oracle d'une indifférence intransigeante. Il y détourne aussi les conseils qu'on lui donne. C'est ainsi qu'à sa mère qui lui suggère de faire du sport (sixième séquence ${ }^{11}$, page 13), le narrateur fait succéder dans son récit, l'écoute à laquelle il se livre, deux fois par semaine, de la retransmission radiophonique des matches du championnat de France de football, dont il dit, par ailleurs, qu'il gagne à être imaginé (séquence 7 , même page). Plus tard, lors de son séjour vénitien, ce seront, le foot encore, à la télé, le cyclisme *verbalisé" (cf. note 3) avec le barman, le tennis impossible et, bien entendu, le seul sport auquel il se livre: les fléchettes dont il finit par blesser Edmondsson.

11 J'appelle -séquences - les portions de texte, toutes numérotées, qui forment le corps du roman; de dimensions fort inégales, allant d'une ligne, voire même d'un mot ( $c f$. la 14 du I : - Maintenant *, p. 17) à plusieurs pages, elles sont au nombre de 40 dans la première partie, "Paris ", 80 dans la deuxième, "L'hypoténuse *, et 50 dans la troisième et dernière, *Paris . encore. Où l'on voit donc trois parties, comme les trois côtés d'un triangle dont *Paris * formerait l'angle droit, - l'hypoténuse * étant, elle, réservée à Venise dont elle est comme le nom effacé, et formée d'un peu moins que la somme des deux autres parties, son carré, cependant, étant quant à lui, au contraire un peu plus que la somme du carré des deux autres. Un peu moins, un peu plus, peut-être faut-il voir là une allusion aux deux infinis pascaliens, mais c'est, en tout cas, une triangulation faussement mathématique qui balise le parcours du narrateur, du fond de sa baignoire parisienne à la ville dans l'eau qu'est Venise, et retour à la baignoire dont l'explicit du roman dit la sortie : - Le lendemain, je sortais de la salle de bain. (II, 50, p. 123). Encore s'agit-il d'une reprise, car le retour est une boucle narrative, si pas diégétique. En effet, la séquence 49 qui précède, reprend alors mot pour mot la séquence $10 \mathrm{du} \mathrm{I}$, la séquence 11 de ce même I se lisant, quant à elle, *Le lendemain, je sortis de la salle de bain. (p. 11). Ce qui empêche la boucle d'être aussi diégétique et de verser ainsi dans une version familière de l'éternel retour c'est, dans la séquence 47 du III, l'allusion faite à la blessure reçue par Edmondsson à Venise: -Sa blessure finissait de se cicatriser" (p. 122). Il est vrai qu'arrive encore la lettre invitant à la réception de l'ambassade d'Autriche... Le trompe-l'ceil ou le paradoxe - topologique. de l'espace narratif est une autre des pratiques qui rattachent Toussaint à Robbe-Grillet. 
16

Un tel détournement qui est aussi, bien entendu, une abstraction (entre autres par la distance de la retransmission radiophonique et de l'épopée nominale), le narrateur l'accomplit aussi sur son espace personnel: d'abord par la baignoire devenue lit mais de méditation et, au commencement l'après-midi seulement, puis par les placards de cette même salle de bain où il déménage une partie de sa bibliothèque, la méditation cédant alors la place à la lecture. Et c'est précisément d'avoir transformé l'espace, de n'avoir pu rester abstrait, d'avoir cédé aux objets, qu'il va se trouver projeté à l'extérieur assez vite, dès la séquence 11 , p. 16: "Le lendemain, je sortis de la salle de bain. .

En vérité, ce qui apparaît d'abord comme un autre coup de tête venant confirmer et résorber le premier, est le résultat d'une série d'opérations qui ne se situent pas qu'au niveau diégétique. Une lecture attentive en fera voir toute la complexité.

Les deux premières séquences (p. 11) s'opposent et se complètent sur un certain nombre de points: de l'une à l'autre, 1) la décision sans volonté de maintien (*Lorsque j'ai commencé à passer mes après-midi dans la salle de bain, je ne comptais pas m'y installer") est devenue au contraire obstination ("mon refus de quitter la salle de bain"), 2) l'acceptation par Edmondsson ("qui

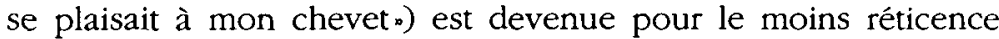
(*Edmondsson pensait qu'il y avait quelque chose de desséchant dans mon refus de quitter la salle de bain "), 3) au niveau du jeu de mot, le liquide (*je coulais là des heures agréables*) est devenu sec (le "quelque chose de desséchant. déjà cité). Enfin, à la mi-journée du séjour de la séquence 1 correspond le travail à mi-temps d'Edmondsson. Et surtout, la baignoire où le narrateur se retranche entre en rapport avec la galerie d'art où Edmondsson travaille, "subvenant aux besoins du foyer ". Car dès lors la peinture va représenter une des structures signifiantes les plus agissantes, avec le sport dont j'ai déjà parlé. Mais si le sport est amené a contrario ( $c f$., ci-dessus, les conseils de la mère), la peinture, elle, coule de source, si je puis dire. C'est ainsi que la troisième séquence, $\mathrm{p}$. 12, la traite de la façon suivante: "Le mur qui me faisait face, parsemé de grumeaux, présentait des craquelures; des cratères ça et là trouaient la peinture terne. "Nous sommes donc passés de l'allusion à un ailleurs où se trouve de la peinture à la description des défauts de celle que le narrateur a sous les yeux, et, banalement, de la peinture (implicite) de la 
galerie d'art ${ }^{12}$ à la peinture industrielle des murs, dévaluation ironique dont les artistes peintres polonais recrutés pour repeindre la cuisine répercuteront la leçon dans la séquence 12: * La galerie d'art dans laquelle travaillait Edmondsson exposait en ce moment des artistes polonais. Comme ils étaient fauchés, Edmondsson m'avait expliqué que l'on pouvait en profiter pour leur faire repeindre la cuisine en les sous-payant. " (p. 16)

Mais nous sommes aussi passés à la catégorie capitale de l'excroissance et du trou. Excroissance des grumeaux, comme un trop-plein d'être, une germination, et trou des cratères, comme un affaissement, une sape. Le surcroît et le manque, l'ajout et la faille ne laissent pas d'être, en sous-main, dans leur combinaison, leur alternance, une cellule génératrice particulièrement agissante. On n'a qu'à penser au rapport, déjà signalé, entre la baignoire et Venise, tel que le narrateur, par son action, le cultive: par son refus de la quitter, il assèche la baignoire, n'y coulant ... que des heures mais veut submerger Venise; il nie ici la propension "naturelle "de la baignoire à être remplie et accentue là une tendance de la ville à être engloutie.

C'est la même rhétorique qui jouera, mais de façon différente, dans la séquence 9, véritable nouud des divers fils jusqu'ici dévidés:

Je portais des vêtements simples [...] Les tissus tombaient avec tant de profit sur mon corps que, tout habillé, je semblais musclé d'une manière fine et puissante. J'étais allongé, détendu, les yeux fermés. Je songeais à la dame blanche, le dessert, boule de glace à la vanille sur laquelle on épanche une nappe de chocolat brûlant [...] D'un point de vue scientifique (je ne suis pas gourmand), je voyais dans ce mélange un aperçu de la perfection. Un Mondrian. Le chocolat onctueux sur la vanille glacée, le chaud et le froid, la consistance et la fluidité. Déséquilibre et rigueur, exactitude (p. 14-15).

Comme si le jeu des vêtements consistait précisément à faire apparaître d'une part ce qui n'est pas, d'autre part ce qu'au contraire ils ont pour fonction de cacher ${ }^{13}$, le motif ici du recouvrement, parti

12 On pourrait objecter qu'une galerie d'art n'expose pas nécessairement que des peintures, mais la suite du texte prouvera bien qu'il n'est pas question d'autres types d'art plastique.

13 Ce que reprendra, en inversant le processus, l'assimilation, plus loin, des poils pubiens d'Edmondsson à : une petite culotte en poil de zébu, en poil de zèbre-(p. 71). 
des grumeaux ${ }^{14}$ du mur, mais en quelque sorte leur contraire (les tissus tombent bien quand le grumeau est, par définition, un défaut; le vêtement dans une baignoire vide est d'une certaine façon un double assèchement quand le grumeau est, au contraire, l'encore liquide) va produire en toute logique la peinture abstraite, " réduction. à des formes et épiphanie de phénomènes d'origine idéelle. Non sans qu'on soit passé par l'évocation de la dame blanche, abstraction justement parce qu'évocation ( «je songeais à la dame blanche") et aidée. ("un aperçu de la perfection"), et grumeau rectifié, puisque "boule" sur laquelle on "épanche" du chocolat. Et la signature de cette "perfection " est Mondrian.

À ce stade, le lecteur un peu averti flaire la mise en abyme: le mélange comme aperçu de la perfection, le mélange et plus encore le contraste, l'opposition violente, la contradiction que fait apparaître la conjonction forcée de deux extrêmes, chaud et froid, consistant et fluide, blanc et noir. Oxymoron structurel, paradoxe du lieu ou de l'objet rendus autres: de la salle de bainchambre à l'hôpital-hôtel, en passant par l'hôtel-terrain de sport. Et si l'hypoténuse c'était aussi cet arc, tendu par l'esprit ou la combinatoire, entre deux exclusives?

En tout cas, la deuxième partie à laquelle elle sert de titre, commence elle aussi par une décision subite ("Je partis brusquement et sans prévenir personne», p. 49) et si un certain effet de dépaysement se renforce paradoxalement du fait que d'abord ni le pays, dont on sait par la présentation seule d'un passeport qu'il est étranger, ni la langue dont on sait seulement qu'elle n'est pas le français, ni enfin la ville dont on n'apprendra qu'assez tard qu'il s'agit de Venise, ne sont identifiés explicitement, c'est d'abord une impression de reprise qui, curieusement, va frapper le lecteur, puisque outre l'ouverture sur la décision subite, celle-ci encore plus étonnante que la première du fait que les faits et gestes du narrateur ont maintenant un passé pour la lecture et qu'ainsi l'absence d'explication fait un peu figure de déni, la séquence 4 de cette deuxième partie présente elle aussi, comme le début du roman, une analepse mais qui, elle, va marquer une différence capitale en installant puissamment, et à travers une discrète allusion à la relativité einsteinienne, la problématique immo-

14 Le deuxième sens de :grumeau •, tel que le définit Le petit Robert, - masse coagulée et gluante dans un liquide ", a peut-être servi de transit du mur à la baignoire. 
bilité-mouvement qui aboutira, page 80 , à la description déjà citée ci-dessus de la dame blanche. Ici, c'est le narrateur luimême, et non son regard, qui en est le siège:

J'avais passé la nuit dans un compartiment de train, seul, la lumière éteinte. Immobile. Sensible au mouvement, uniquement au mouvement, au mouvement extérieur, manifeste, qui me déplaçait malgré mon immobilité, mais aussi au mouvement intérieur de mon corps qui se détruisait, mouvement imperceptible auquel je commençais à vouer une attention exclusive, qu'à toutes forces je voulais fixer. (p. 51)

Misère de l'homme voué au mouvement jusque dans son corps, misère que le narrateur a tenté de fuir cette fois dans la chambre immobile d'une ville flottante et dont il demandera plus loin à Edmondsson qui l'a rejoint à Venise de le *consoler": "Je lui demandais de me consoler. D'une voix douce, elle me demandait de quoi je voulais être consolé. Me consoler, disais-je. Mais de quoi, disait-elle. Me consoler, disais-je (to console, not to comfort)." (p. 86-87) Suit la citation, en anglais, du célèbre texte de Pascal sur le divertissement (pensée 139): *But when I thought more deeply, and after I had found the cause for all our distress, I wanted to discover its reason, I found out there was a valid one, which consists in the natural distress of our weak and mortal condition, and so miserable, that nothing can console us, when we think it over (Pascal, Pensées). "(p. 87) ${ }^{15}$

On n'aura garde de passer ici sous silence la très curieuse typographie, contraire à tous les usages, de cet extrait. Avant la citation de Pascal, l'anglais entre parenthèses n'est pas, comme il le devrait, en italique, mais dans le texte de Pascal, qui devrait, lui, en toute logique, être entièrement en italique, le mot qui fait l'objet de la question de traduction soulevée par le narrateur, l'est, lui. C'està-dire que le texte de Pascal - véritable générateur du projet de tout le roman, cela semble évident, et générateur jusque dans son écriture, elle aussi étrangement tautologique ou infimement variationnelle, sorte de quiétisme contemplatif de la lettre - est l'objet d'un double détournement: traduit, et dans une langue triplement

15 - Mais quand j'ai pensé de plus près, et qu'après avoir trouvé la cause de tous nos matheurs, j'ai voulu en découvrir la raison, j'ai trouvé qu'il y en a une bien effective, qui consiste dans le malheur naturel de notre condition faible et mortelle, et si misérable, que rien ne peut nous consoler, lorsque nous y pensons de près.= Ibid., note 10, p. 109-110. 
étrangère (à son auteur, bien sûr, c'est l'évidence de la traduction, mais aussi au lecteur, et c'est alors un véritable renversement puisque ce lecteur, le narrateur, parle la même langue que l'auteur traduit, comme s'il leur fallait un tiers étranger pour communiquer entre homophones, et enfin au pays même où le livre se trouve ${ }^{16}$ ), il se voit typographiquement pris comme corps d'une citation du texte de Toussaint, comme illustration d'un problème de traduction $^{17}$, et donc l'italique est ici un souligné. Un souligné qui fait à Blaise Pascal le coup intertextuel du "signifiant de contact". Il y a aussi, décidément, du Roussel dans ce Toussaint-là. Le côté "échiquéen " d'une écriture très stratégique et pleine d'arrière-pensées...

Ce signifiant de contact est d'ailleurs un peu l'équivalent de ce qu'est pour le narrateur le jeu de fléchettes: la précipitation du sens dans la téléologie apparemment imparable du signifiant, le triomphe du référent comme étymon et aboutissement, alpha et oméga, justification du parcours et dépassement de la contingence. Je l'ai dit, ce jeu de fléchettes représente une version moderne de l'air de jazz chanté par la négresse dans La nausée. Voyons un peu.

"Lorsque je jouais aux fléchettes, j'étais calme, détendu. Je me sentais apaisé. Le vide me gagnait progressivement et je m'en

16 Si l'on écarte l'interprétation hyperréaliste - le petit détail que son absurdité même fait vrai - ou réaliste - dans un hôtel d'une ville internationale, on peut effectivement faire ce genre de trouvaille - ce Pascal anglais lu par un Belge en Italie illustre plutôt l'irrépressible mouvance du signifiant lui-même, Pascal, pas plus que Rome n'est dans Rome, n'est dans Pascal, et rien ne - console * du malheur de son parcours qui forme notre vie de la trace mélancolique qui nous meut. Pascal, ainsi traité, vient rejoindre les noms des coureurs cyclistes déjà mentionnés et la rencontre qui fait pendant à cette scène, d'un touriste soviétique à l'aéroport de Venise: "Après un bref tour d'horizon de l'histoire italienne du vingtième siècle (Gramsci, Mussolini), nous demandâmes d'autres bières. Puis, passant à l'histoire de son pays; sujet plus délicat en raison du joug, nous dîmes Kroutchev, Brejnev. Je citai Staline. Il but pensivement une gorgée de bière... (p. 119). Les noms propres que l'on s'échange ainsi sont de simples surfaces de contact, des signifiants purs. Pascal *extextualisé* - comme on dit exterritorialisé - remplit un peu la même fonction: il est, signifiant et signifié confondus dans l'indécision abstraite de la traduction, le signe pur de la * consolation. que le narrateur réclame d'Edmondsson.

17 Nul doute que dans l'atténuation, l'oblique, qui caractérisent la manière de Toussaint, ce problème de traduction, soulevé devant la femme aimée pour justifier l'irréductibilité d'une demande, ne corresponde à ce qu'un auteur plus pompeux claironnerait dans l'absurde: incommunicabilité, solitude indépassable qui font *le malheur naturel de notre condition * 
pénétrais jusqu'à ce que disparût toute trace de tension dans mon esprit. Alors - d'un geste fulgurant - j'envoyais la fléchette dans la cible" (p. 83). Au-delà de la référence explicite à l'archer zen, c'est le "divertissement "pascalien qui ici s'affirme, de toute la force de son sens retrouvé, de son but, de sa cible, fût-elle futile, et d'autant plus qu'elle l'est. Consolation véritable, le jeu de fléchettes engouffre aussi le social dans un solipsisme vertigineux: non content de jouer seul, le narrateur va jusqu'à s'inventer une ligue internationale, jouant tour à tour " au nom " d'équipes nationales (comme le football, ce jeu "gagne à être imaginé") et faisant en sorte que son pays, la Belgique, gagne la finale.

Nationaliste, identitaire, opérant une "concentration" du sujet ( Dès la première série de lancers, mon peuple, très concentré, prit facilement l'avantage sur ces maladroits de Français" (p. 84). On aura noté l'humour de ce "très concentré ": "concentré", son "peuple" ne saurait l'être plus qu'ainsi réduit à lui), la fléchette c'est aussi la perfection de l'immobilité enfin atteinte, mais, pour ainsi dire, de toute éternité. Et la séquence qui suit immédiatement celle de la finale né laisse pas d'être particulièrement révélatrice puisque revoici Mondrian et bientôt, menacée en sous-main, Edmondsson:

Ce qui me plaît dans la peinture de Mondrian, c'est son immobilité. Aucun peintre n'a voisiné d'aussi près l'immobilité. L'immobilité n'est pas l'absence de mouvement, mais l'absence de toute perspective de mouvement, elle est mort. La peinture, en général, n'est jamais immobile. Comme aux échecs, son immobilité est dynamique. Chaque pièce, puissance immobile, est un mouvement en puissance. Chez Mondrian, l'immobilité est immobile. Peut-être est-ce pour cela qu'Edmondsson trouve que Mondrian est chiant. Moi, il me rassure. Une fléchette dans la main, je regardais la cible accrochée sur le battant de l'armoire, et je me demandais pourquoi cette cible, plutôt qu'à Jasper Johns, m'avait fait penser à Edmondsson (p. 84).

Ainsi, l'aimmobilité immobile" de Mondrian est-elle la perfection du sens, perfection que la dame blanche ne faisait qu'évoquer, et qui s'obtient par le dépassement du contraste, de l'oxymoron fondateur, dans un aboutissement qui est aussi une résorption. Cette dialectique de la mort comme cible n'est pas sans évoquer, elle aussi, le Heidegger de l'nêtre-pour-la-mort" (Sein zum Tode), dont on se souviendra qu'il désigne non seulement, bien sûr, notre indépassable destin mais aussi le but, la mesure (la "ciblen?) de l'authenticité de l'étant aux prises avec 
cette acceptation nécessaire et dynamisante. La mort est ainsi la * tautologie variationnelle . de l'étant: un espace immobile où creuser son méandre. On pourrait sans doute en dire autant, qui ne le voit?, de l'écriture, le traitement réservé à la tautologie pascalienne le dit assez clairement.

Mais, en ce qui concerne le narrateur, ce "divertissement" qui porte en lui les germes de son dépassement (serait-ce la "foi " pascalienne?) retombera justement dans l'insignifiance du simple jeu quand Edmondsson tentera de l'en divertir, aimable principe de réalité qui opère un détournement de cible. Déjà, on l'a vu, la cible évoquait, plus que l'artiste américain, Edmondsson. Par ailleurs, la séquence qui suit celle de l'immobilité de Mondrian, évoque les cauchemars du narrateur, peuplés d'^images obsédantes de cibles" (p. 85) et organisés selon une dialectique que nous commençons maintenant à bien connaître: *un tourbillon qui m'englobe et m'emporte en son centre, par exemple, ou des lignes droites placées devant mes yeux dont je tâche infiniment de modifier la structure, remplaçant un segment par un autre, procédant à des corrections sans fin pour les épurer. (Ibid.). Du tourbillon à l'épure et du mouvement à l'immobilité, de la passivité à l'activisme et de la nature métaphorique à la culture géométrique, c'est toute l'obsession de salut du narrateur qui se trouve ainsi une légende. Et c'est par un véritable sacrifice qu'Edmondsson viendra suturer la schize où le sujet indéfiniment s'enfonçait : * Edmondsson me trouvait oppressant. Je laissais dire, continuais à jouer aux fléchettes [...] Debout devant la fenêtre, Edmondsson me regardait fixement. Elle me demanda une nouvelle fois d'arrêter. Je lui envoyai de toutes mes forces une fléchette, qui se planta dans son front. Elle tomba à genoux par terre." (p. 88)

Edmondsson devenue cette cible que le texte déjà annonçait, tout peut repartir en sens inverse, sorte de résorption narrative qui va ramener le narrateur vers Paris, vers la salle de bain encore et, une fois de plus, la sortie de la salle de bain, mais une sortie cette fois-ci finale puisqu'elle clôt le roman. Mais tout ce processus sera l'effet d'une contagion, celle du bouc émissaire (Edmondsson) lorsqu'il renvoie le mouvement social qui l'a produit vers ceux qui l'ont déclenché, inversant ainsi la direction. En effet, dès les premières pages de la troisième partie, le narrateur, après avoir raccompagné Edmondsson au train pour Paris, se sent fiévreux, va passer des radiographies du front et du nez, finit par aboutir lui aussi à l'hôpital. Puis, de fil en aiguille, d'invitation à 
souper chez le médecin à une partie de tennis avec lui et sa femme, le narrateur va retrouver la fixité du regard d'Edmondsson au fond de ses propres yeux, dans l'espace sanitaire des toilettes du club: "Debout devant le miroir rectangulaire des toilettes, je regardais mon visage qu'éclairait une lampe jaune derrière moi [...] Je regardais mon visage ainsi divisé par la lumière, je le regardais fixement et me posais une question simple. Que faisais-je ici?" (p. 116). Passé -par l'hôtel où il ne réside plus, pour demander s'il a du courrier, puis rentré dans sa chambre à l'hôpital qu'il a transformé en hôtel et y trouvant un autre locataire, il décide enfin de rentrer à Paris. Puis dans sa salle de bain. La boucle est bouclée mais le développement des variations en forme d'épure ne s'est pas complètement résorbé : il reste cette petite cicatrice sur le front d'Edmondsson, cette petite cicatrice qui tel un clinamen déplace légèrement le parcours et fait que, coïncidant alors avec la sortie du livre, la deuxième sortie de la salle de bain est la bonne.

La réticence, au contraire, commencera non par une occupation obstinée et déraisonnable de l'espace privé mais par une errance, l'arrivée dans l'espace privé-public d'un hôtel (italien encore) mais vue sous forme d'analepse, à partir d'une durée indistincte, dans sa précision même - "Ce matin,. il y avait un chat mort dans le port, un chat noir qui flottait à la surface de l'eau (incipit, p. 11) - et se terminera par le passage d'un navire, :qui gliss(e) immobile à la surface de l'ẹu et qui finit par disparaître très lentement derrière lî̀le de Sasuelo" (p. 159).

La structure narrative en boucle de La salle de bain aboutira à une structure plus complexe, "ondulatoire", avec La réticence où un certain nombre d'analepses s'articulent à une variation sur le cadavre du chat qui semble bien un autre discret salut à RobbeGrillet, celui des Gommes cette fois, où l'on peut lire dans les nombreuses descriptions de l'eau du canal ou de celle de l'aquarium du café la même fonction, scripturalement génératrice de fictions ou diégétiquement productrice de "fantasmes" du narrateur, que celle remplie ici par le cadavre du chat. Parmi les plus révélatrices, celle-ci qui pourrait servir d'exergue à La réticence:

Les miettes éparses, les deux bouchons, le petit morceau de bois noirci: on dirait à présent comme une figure humaine, avec le bout de pelure d'orange qui fait la bouche. Les reflets du mazout complètent un visage grotesque de clown, une poupée de jeu de massacre. 
Ou bien c'est un animal fabuleux: la tête, le cou, la poitrine, les pattes de devant, un corps de lion avec sa grande queue, et des ailes d'aigle. La bête s'avance d'un air gourmand vers une proie informe étendue. un peu plus loin. ${ }^{18}$

Bien entendu, l'inscription ici de la sphynge grecque renvoie à CEdipe et à l'intertexte du roman, alors que le roman de Toussaint, lui, renvoie précisément à cet intertexte-ci par au moins deux traits (outre la mise en abyme, commune au deux textes, d'une cellule génératrice capitale): le motif de la nourriture et la famille des félidés, le chat de Toussaint, lui, portant en outre lui aussi un discret motif fabuleux ou étrange dans la mesure où il est mort d'avoir franchi la frontière des espèces en tentant de s'emparer d'un appât à poisson.

Quoi qu'il en soit, plus spécifiquement, de ce dernier roman publié de Toussaint, qu'il n'est plus temps de traiter ici, il reste que l'œuvre apparaît étonnamment systématique et conséquente, chacun des romans jouant lui-même le rôle d'une variation dans un ensemble dont La salle de bain semble bien avoir fourni les paramètres fondateurs : une interrogation "ontologique" qui porte à la fois sur l'énoncé et sur l'énonciation, sur l'écriture et la fiction qu'elle impulse et dont l'inquiétante familiarité des comportements sociaux qui s'y trouvent décrits provient de leur "nature" de mouvements corpusculaires qui, partant, n'ont nulle raison à donner, une imperturbable acceptation régissant dès lors les rapports entre les individus, comme elle marque l'écriture du sceau de l'évidence tranquille de sa pure effectuation.

Et si cette interrogation ontologique, cet humour imperturbable, cette déviation tranquille scellaient, sous le signe de Lucrèce et du matérialisme antipositiviste de la lettre, l'alliance longtemps tenue (à peine) secrète du Nouveau Roman et de la Pataphysique?

18 Les gommes, Paris, Éditions de Minuit et U.G.E., coll. «10/18*, 1962 [1954], p. 37. 